pag

Business School

WORKING PAPER SERIES

\begin{tabular}{c|l} 
Working Paper & $\begin{array}{l}\text { Threshold Preferences and the } \\
\text { Environment }\end{array}$ \\
$2013-015$ & $\begin{array}{l}\text { Ingmar Schumacher } \\
\text { Benteng Zou }\end{array}$
\end{tabular}

http://www.ipag.fr/fr/accueil/la-recherche/publications-WP.html

IPAG Business School

184, Boulevard Saint-Germain

75006 Paris

France

IPAG working papers are circulated for discussion and comments only. They have not been peer-reviewed and may not be reproduced without permission of the authors. 


\title{
Threshold Preferences and the Environment
}

\author{
Ingmar Schumacher* Benteng Zou ${ }^{\dagger}$
}

June 23, 2013

*IPAG Business School, 184 boulevard Saint-Germain, 75006 Paris, France, and Department of Economics, Ecole Polytechnique, Paris, France. Tel.: +352 621264575. E-mail: ingmar.schumacher@ipag.fr

${ }^{\dagger}$ CREA, Université du Luxembourg, 162A avenue de la Faïencerie, L-1511 Luxembourg. Tel:+352 4666446622. E-mail: benteng.zou@uni.lu.

We are grateful for comments from Cuong Le Van and Agustin Perez Barahona. The second author appreciates the support of COST Action IS1104 "The EU in the new economic complex geography: models, tools and policy evaluation". 


\begin{abstract}
In this article we study the implication of thresholds in preferences. To model this we extend the basic model of John and Pecchenino (1994) by allowing the current level of environmental quality to have a discrete impact on how an agent trades off future consumption and environmental quality. In other words, we endogenize the semi-elasticity of utility based on a step function. We motivate the existence of the threshold based on research from political science, from arguments based on regulation and standards, cultural economics as well as ecological economics.

Our results are that the location of the threshold determines both the potential steady states as well as the dynamics. For low (high) thresholds, environmental quality converges to a low (high) steady state. For intermediate levels it converges to a stable p-cycle, with environmental quality being asymptotically bounded below and above by the low and high steady state. We discuss implications for intergenerational equity and policy making.

As policy implications we study shifts in the threshold. Our results are that, in case it is costless to shift the threshold, it is always worthwhile to do so. If it is costly to change the threshold, then it is worthwhile to change the threshold if the threshold originally was sufficiently low. Lump-sum taxes may lead to a development trap and should be avoided if there are uncertainties about the threshold or the effectiveness of the policy.

JEL classification: Q28, Q56.

Keywords: thresholds, endogenous preferences, environmental quality, policy intervention.
\end{abstract}




\section{Introduction}

It is public opinion that drives governments' actions, decisions, laws, changes the political agenda and sometimes even its direction. But public opinion tends to be sticky and requires enough pressing issues for it to change. However, if sufficiently pressing problems are identified, then preferences undertake radical changes. This has been observed to be especially true for concerns directed to the environment. In this respect, Erskine (1972) noticed that "[a] miracle of public opinion has been the unprecedented speed and urgency with which ecological issues have burst into American consciousness." She then continues by saying that "[t]he environment does approach top priority today for expanded governmental spending in the opinion of the citizenry." In this article we model this radical change in the preferences and study its effects. To do so, we introduce the idea of threshold preferences to investigate the impact of discrete changes to preference on the trade-off between consumption and the environment. In other words, we endogenize the semi-elasticity ${ }^{1}$ of utility based on a step function. ${ }^{2}$ Specifically, we suggest that if environmental quality is below a threshold then this increases agents' semi-elasticity of environmental quality in utility. In contrast, if agents are faced with a state of abundant environmental quality above that threshold, then their preferences are directed relatively less towards the environment, in the sense that the agent's semi-elasticity of environmental quality in utility will be low. In other words, we suggest that the current level of environmental quality has a discrete impact on how an agent trades off future consumption and future environmental quality.

The point that we develop here is that preferences should be viewed as being endogenous, but changes to the preferences require sufficiently strong impulses. Consequently, the model that we propose here has some relation to the class of models based upon lexicographical preferences (Sen (1970); Gelso and Peterson (2005)). Lexiographical preferences are an interesting modeling approach since they allow to deal with the crossing

\footnotetext{
${ }^{1}$ The semi-elasticity of a function $f(x)$ is defined as $f^{\prime}(x) x$.

${ }^{2}$ Prieur and Bréchet (2013) endogenize the semi-elasticity of utility via education expenditure, while Goulao and Pérez-Barahona (2013) endogenize it via health capital.
} 
of thresholds that are tacitly important for a society. This is relevant especially for the environmental domain. Specifically, actions devoted to environmental protection tend to be undertaken when situations are dire or pressing enough. As an example, the Cuyahoga River in the USA was so polluted that it caught fire several times. In consequence of this extreme pollution the Environmental Protection Agency was established, as well as a multitude of water quality regulations.

Our modeling approach is based upon the overlapping generations model of consumption and the environment by John and Pecchenino (1994). We extend their work by introducing the idea of threshold preferences ${ }^{3}$. As we shall show, our model of threshold preferences has further effects than a mere acceleration one. Crucially, the position of the threshold determines the dynamics and implications of this model. We show that for sufficiently high threshold levels, which would, for example, be the case for an ecologicallyoriented society, our model predicts that this society will grow monotonically towards a high level of environmental quality. Instead, societies will be likely to converge to a low level of environmental quality if they only place priority on the environment in dire situations. This would be the case if the threshold is sufficiently low. However, for mixed societies, namely those that are in neither of these two extreme cases, we find that environmental quality will not converge to any steady state. Instead, when environmental quality is bad, then their preferences will be directed relatively more towards environmental quality, and consequently the state of the environment will improve. Once it has improved sufficiently (i.e. crossed the threshold), then the society's preference will shift back towards a more consumption-oriented one, implying less effort directed towards the environment. This will therefore induce a never-ending cycle. One could say this occurs simply because societies in this case are not sufficiently far-sighted and their reference point of environmental quality, or their status quo, essentially only depends on today's environmental quality. Or, in a slightly different interpretation, society's preferences lack sufficient consistency in a sense that they are too easily shifted by a reference point.

Though we take a black-box approach to the threshold in the model, we provide several

\footnotetext{
${ }^{3}$ We came across this idea due to an article by Goulao and Pérez-Barahona (2013).
} 
ways in which the underlying mechanics can be derived. To do so, we freely borrow from previous works in political science, regulation and standards, cultural economics as well as ecological economics. These approaches do not provide a full-fledged micro-foundation for our threshold, but they can nevertheless help in supporting our reduced-form threshold model based on existing research from different fields.

We also show that there is a certain complementarity between the environmental quality that an individual or a society faces today, and the one that he or she is confronted with in the future. Thus, in a sense, our article borrows from the literature on intertemporallydependent preferences (Ryder and Heal (1973), Wan (1970)). Specifically, in our context this means that environmental quality today impacts how the individual or society values future environmental quality, and thereby affects the trade-offs between polluting and abating behavior. In this respect, an approach that is related to ours is the one presented in Schumacher and Zou (2008). In that article we pursue the idea that agents perceive the state of environmental quality not based on the actual level, but with reference to the status quo. In other words, agents cannot fully relate to the environment as it was before they were born, and are thus more strongly concerned with changes in the environment than with the level itself. The main result in that article is that, for a large set of parameters, the model would generate complex dynamics which would lead to violations of standard criteria of intergenerational equity. In the current work we introduce a different yet complementary approach by suggesting that changes to the preferences only occur once a certain threshold has been crossed. The implication of this threshold is again that cycles are possible and therefore standard criteria of intergenerational equity will be violated, but the underlying reasons are different. In Schumacher and Zou (2008) cycles occurred because agents got habituated to pollution, while in the current approach agents change their preferences if society feels sufficient environmental pressure.

Our work also links to the article by Prieur and Bréchet (2013) as well as Goulao and Pérez-Barahona (2013). In Prieur and Bréchet (2013), the authors endogenize the semi-elasticity of environmental quality via human capital, and call this expenditure in environmental awareness. They study whether education in environmental awareness 
may lead to sustained growth, or induce society to converge to a steady state. These authors show that, if environmental awareness is sufficiently sensitive to human capital, then society may not converge to the sustained growth equilibrium any longer but only to the steady state. Thus, the authors show how an endogenous environmental awareness can lead to a qualitative change in the dynamics. However, they obtain this by allowing for a balanced growth path, while we solely focus on a steady state economy. Also, we obtain much richer dynamics, and focus on a different source of endogeneity. In the article Goulao and Pérez-Barahona (2013) the authors also endogenize the semi-elasticity of utility, but this time they study the impact of health capital on survival probabilities. The main mechanism in common with us is the authors' focus on a step-function. For high health capital, the semi-elasticity (probability of survival) of health in utility is high, while for low health capital this semi-elasticity takes a low value. These authors also find the existence of two stable steady states in their case. In contrast, our dynamics are much richer, and the reason for this is that, in our case, the endogenous feedbacks are more complex.

Our work also links to the class of endogenous preferences (Bowles (1998)) and cultural traits (Bisin and Verdier (2001)), even though we neither explicitly model the underlying cultural dynamics nor group heterogeneity. However, we show how a simple model of endogenous cultural traits can result in the type of utility function that we use in our model. This approach is in line with Schumacher (2009). In contrast to our current work, the modeling approach in Schumacher (2009) lacked an endogenous, explicit dynamic choice mechanism. In this article we present one way in which this choice mechanism can be modeled. While the results in Schumacher (2009) are based on an implicit decision progress, those presented here are based on an explicit, dynamic one.

Our results are that the location of the threshold determines both the potential steady states as well as the dynamics. For low (high) thresholds, environmental quality converges to a low (high) steady state. For intermediate levels it converges to a stable p-cycle, with environmental quality being asymptotically bounded below and above by the low and high steady state. We discuss implications for intergenerational equity and policy making. As 
policy implications we study shifts in the threshold. Our results are that, in case it is costless to shift the threshold, it is always worthwhile to do so. If it is costly to change the threshold, then it is worthwhile to change the threshold if the threshold originally was sufficiently low. Lump-sum taxes may lead to a development trap and should be avoided if there are uncertainties about the threshold or the effectiveness of the policy.

The paper is organized as follows. In section 2 we motivate our model through relying on studies from political science, from cultural economics, and ecological economics. We show how each of these disciplines can imply the extension to the utility function that we introduce here. Section 3 introduces the model and studies its dynamics and steady state properties. Here we also discuss the results and relate it to John and Pecchenino (1994) and Schumacher (2009). In section 4 we discuss lessons for intergenerational equity and under what circumstances the model predicts convergence to a disastrous level of environmental quality. We look into possible policies in section 5. In section 6 we conclude.

\section{Background}

In this part we borrow extensively from different disciplines in order to give a foundation to the model extension of John and Pecchenino (1994) that we propose in the next section. Those disciplines are political science, regulation and standards, cultural economics and ecological economics. We review how some approaches or results of the established literature can be viewed within our setting as outlined above.

\section{$2.1 \quad$ Political science}

Downs (1972) describes the "issue-attention cycle", which proceeds in five stages. In stage 1, which he calls "the pre-problem stage", some undesirable social condition exists

without being fully incorporated into the public opinion. In stage 2, dubbed the stage of "alarmed discovery and euphoric enthusiasm", a drastic worsening of the social condition leads to an euphoric public that wants to take care of the problem. His third stage, the 
"realizing the cost of significant progress" stage, is then followed by the stage of "gradual decline of intense public interest" followed by a so-called "post-problem" stage. In this fifth stage, potentially new institutions or laws that were originally designed to treat the social condition continue to exist and may still have some impact.

In other words, a slowly deteriorating (environmental) condition may be unnoticed by the general public unless a certain threshold is crossed, or a drastic worsening of the situation calls for public attention. In that case preferences get strongly directed towards this problem. Whether the third stage turns out to be an important driver of continuous public interest is certainly depending on the situation at hand. However, what Downs (1972) seems to say is that this public attention gets directed to an immediate problem, and for some reason fades away again after some while. This may be, as Downs suggests, because costs are too high, but it may also be because the problem has been resolved to a sufficient extent. What this suggests, nevertheless, is that there is a point at which attention gets directed towards a problem, while attention is directed elsewhere if the problem cannot be resolved or is taken care of. Thus, this may lead to never-ending cycles of issue-attention. We now provide two examples for this issue-attention cycle, followed by a small model that may explain how voters' preferences transcendent into government preferences and thereby induce a threshold effect that may lead to the issue-attention cycle.

Example 1. As an example from financial economics we propose the Glass-Steagall Act, which nicely fits the issue-attention cycle. In order to separate commercial banking and investment banking, the union of which was believed to be one source of the 1929 financial crisis, the Glass-Steagall Act was passed in the US in 1933. In Kennedy's (1973) opinion, this bill came about only due to strong public pressure after the crisis. In 1999, President Clinton decided to suspend the Act, allowing commercial banks and investment banks to re-unite. Only ten years later, as a consequence of the 2008 financial crisis, public opinion is demanding a move back to 'narrow banking', which would basically imply the re-introduction of a version of the Glass-Steagal Act. What we thus see here is that a certain threshold needs to be crossed before public attention gets mobilized. In 
the present example this threshold is given by the discrete move from a non-crisis to a crisis situation. ${ }^{4}$

Example 2. Another example comes directly from politics. In the aftermath of Fukushima, 2011, the CDU, the ruling party in Germany at that time, decided to shut down most atomic power plants in Germany. This decision reflected a complete turnaround in this party's attitude to atomic power, since just a few years before they repealed a law that required several German atomic power plants to shut down. Consequently, this extreme event led to a 180-degree turnaround in policy.

We present a simple model that provides one way to justify the approach taken in the next section. Let us assume that utility takes the form $v_{i}=\theta_{i} \log \left(E_{o}+E\right)-E$. Here, utility is increasing in the public good $E_{o}+E$, where $E_{o}$ is the current level of environmental quality, but there is a unit cost that each individual needs to bear in order to increase $E$. Preferences $\theta_{i}$ are distributed over $\theta_{i} \in\left[\theta_{\min }, \theta_{\max }\right]$. Individual $i$ will vote for an increase in the public good $E$ if $\theta_{i}>E_{o}+E$. The government adopts the preferences of the median voter, which are given by $\theta_{m}=E_{o}+E$. Thus, for $E_{o}>\theta_{\max }$, the government will have preference type $\theta_{\text {min }}$, while for $\theta_{\min } \geq E_{o}$, the government's type will be $\theta_{m}>\theta_{\min }$. As a consequence, the current level of environmental quality affects the preferences that the government adopts. If the initial environmental quality is sufficiently high, then the preferences of the policy maker will include environmental quality only marginally, while for low levels of environmental quality, these preferences will be more strongly directed towards environmental quality. This, thus, may explain why thresholds in the utility function, here a policy maker's, may occur.

\subsection{Regulations and standards}

The government has several methods to address environmental problems. Apart from economic incentives like taxes, subsidies or cap-and-trade, the policy maker can also

\footnotetext{
${ }^{4}$ The resulting regulation-deregulation cycle has been shown to exist in an empirical study by Lee and Schumacher (2011). This article shows that mainly crises lead to changes in attitude and thus regulation, while the authors find that in times of stability attitudes turn around and induce de-regulations.
} 
provide prescriptive controls, like technology standards (e.g. best available technology), performance standards, emission or ambient standards, which are more of the commandand-control type. The standards are then based on some analyzes like cost-benefit studies, precautionary principles, health objectives or simply a policy target. This is laid out e.g. in Section 108 of the Clean Air Act demanding that "[a]ir quality criteria for an air pollutant shall accurately reflect the latest scientific knowledge useful in indicating the kind and extent of all identifiable effects on public health or welfare which may be expected from the presence of such pollutant in the ambient air, in varying quantities." Based on these criteria and health considerations, article 5 (b) of the EU Directive 2001/81/EC requires that, in member states, "the ground-level ozone load shall not exceed an absolute limit of 2.9 ppm.h in any grid cell." In case a pollution threshold is violated, then article 19 of the EU Directive 2008/50/EC demands that "Member States shall take the necessary steps to inform the public by means of radio, television, newspapers or the Internet."

This leads to the common belief that, after a standard has been imposed, any pollution level above the standard is safe and fine, while any pollution level below the standard is worrisome and needs to be addressed. As a result, this leads to a threshold effect. ${ }^{5}$

It is certainly unreasonable to belief that any level of pollution above the standard is safe while any level of pollution below the standard requires immediate policy action. In reality, health effects are more likely to be depending on the level of pollution in a continuous way. Nevertheless, the simple fact that a standard is imposed leads to inaction for pollution levels above the standard and action below the standard.

If one were to model this, then in a slightly generalized way one could say that the individual's utility function depends on the threshold. For environmental quality above the threshold, the individual has little attention directed towards environment quality, or obtains a low marginal utility from environmental quality, while for environmental

\footnotetext{
${ }^{5}$ Standards are not always applicable, as the EU Directive 2008/50/EC suggests: "Fine particulate matter $(\mathrm{PM} 2,5)$ is responsible for significant negative impacts on human health. Further, there is as yet no identifiable threshold below which PM2,5 would not pose a risk. As such, this pollutant should not be regulated in the same way as other air pollutants." In these cases, depending on the way the pollutant is finally regulated, threshold effects may or may not occur.
} 
quality below the threshold the individual concern increases with a discrete step, and the individual obtains a high marginal utility from environmental quality. We model this mechanism in the next section.

\subsection{Cultural economics}

Cultural economics plays an increasing role for modeling endogenous preferences. Especially for environmental problems, this approach promises to be useful. In this respect, Linton (1963 (1940 reprint)) notes that (p. 466) “... [c]ultures are adaptive mechanisms and as such represent a response to the needs of our species." Empirical evidence suggests that cultural influences cannot anymore be neglected by economists. For example, Graumann and Kruse (1990) conclude that social relations form attitudes and values. More specifically, Villacorta et al. (2003) find that children's environmental self-regulation is influenced by their parents' self-regulation. Also, Olli et al. (2001), relying on a Norwegian social survey, conclude that the social network drives environmental behavior. Thus, preferences should be viewed as being endogenous and culture seems to play a crucial role.

We now present a simple cultural dynamics mechanism that can explain the modelling approach of the preferences that we use in the next section. This mechanism is developed and studied in Schumacher (2009) and derives from the cultural dynamics introduced in Bisin and Verdier (1998) and (2001). Preferences are ordered over $\theta_{t} \in[0,1], t=1,2, \ldots$, with $\theta_{t}=0$ denoting brown ones and $\theta_{t}=1$ implying green ones. We assume that the children adapt their preferences either from their green (brown) parents with a probability $q \in(0,1)$, or are influenced by someone with either of the two preference traits. The probability $q(E)$ takes the form $q^{\prime}(E)<0$, meaning that the larger is environmental quality $E$ the lower is the probability to adapt green preferences. A child born by brown parents keeps the brown preference trait with probability $\bar{q}$. As a consequence, children 
change their preferences based on this reduced-form equation ${ }^{6}$

$$
\dot{\theta}_{t}=\theta_{t}\left(1-\theta_{t}\right)(q(E)-\bar{q}) .
$$

There are three potential equilibrium points, given by $\theta=0, \theta=1$, and $q(E)=\bar{q}$. If $q(E)>\bar{q}$ then we converge to $\theta=1$, while for $q(E)<\bar{q}$ we converge to $q=0$. Assume that there are no immediate feedbacks from $\theta$ to $E$, then this implies that only $\theta=1$ and $\theta=0$ are asymptotically stable. Thus, we end up with a utility function that, depending on the level of environmental quality, is either green or brown. The threshold at which this discrete change occurs is where $E=q^{-1}(\bar{q})$. We thus may conclude that cultural dynamics can give rise to thresholds in the utility function.

\subsection{Ecological Economics}

As much of the literature in Ecological Economics has pointed out, monetary values are but one aspect of environmental values (Lockwood (1996); Gowdy (1997); Splash (2000)). Other factors like ethical concerns, basic needs, or culture-specific wants led many ecological economists to the conclusion that lexicographical preferences should supersede the neoclassical paradigm. Popularized by Georgescu-Roegen (1954), these lexicographical preferences imply that substitution between inputs in the utility function is impossible since they give an absolute priority to one good over another. For example, the protection of the habitat of endangered species often receives absolute priority over economic developments of that area. Similarly, once a species is in danger of extinction, then substantial efforts will be undertaken to prevent this from happening.

Indeed, there is now ample empirical evidence supporting the existence of lexicographical preferences. For example, Stevens et al. (1991) found that around a quarter of the individuals in their US study about wildlife preservation hold lexicographical preferences. A similar percentage has been identified as holders of lexicographical preferences in a survey on wetlands preservation by Spash (2000). In an experiment on improvements

\footnotetext{
${ }^{6}$ The derivation of this equation can be found in Schumacher (2009).
} 
of rural landscape attributes, Campbell et al. (2008) find that discontinuous preferences help explain the magnitude and robustness of willingness to pay estimates.

We build upon these empirical approaches and in what follows, we shall adapt a type of lexicographical preferences, but to a limited degree. Instead, what we want to do is to add a lexicographical flavor to the standard neoclassical modeling structure. Figure 1

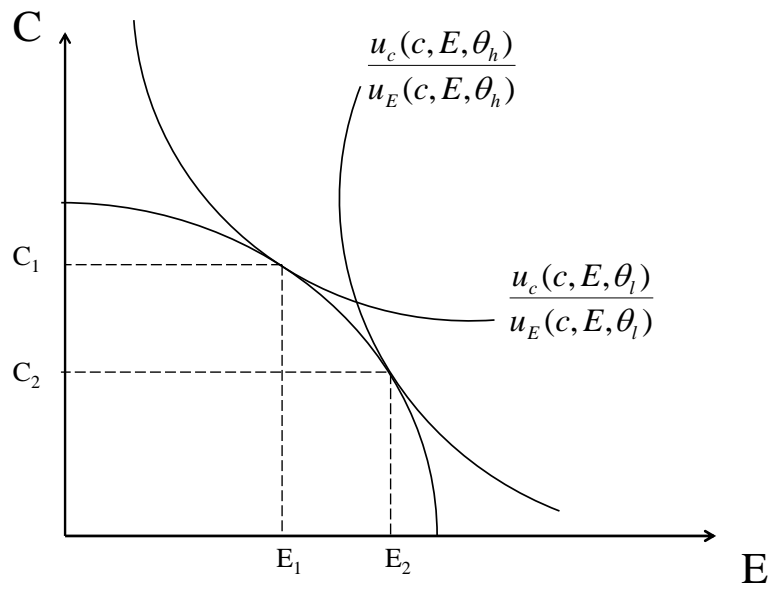

Figure 1: Effect of $\theta_{H}$ and $\theta_{L}$

shows what we have in mind. Assume we have a situation where $E_{t}>\widehat{E}$. Thus, current environmental quality $E_{t}$ is above a certain threshold $\widehat{E}$. In this case the marginal rate of substitution between consumption and environmental quality will, for a given budget constraint, induce society to choose the optimal bundle $\left\{C_{1}, E_{1}\right\}$. However, assume now that - still given the same budget constraint and the same level of environmental quality, the threshold $\widehat{E}$ were to be lower. In this case society would give a heightened, near absolute, priority to the environment over consumption, which would be reflected by a change in the marginal rate of substitution. In this case society would be inclined to choose bundle $\left\{C_{2}, E_{2}\right\}$, where $C_{1}>C_{2}$ but $E_{1}<E_{2}$.

A utility function that could take this threshold event into account would have a 
discontinuity at the point of threshold. This discontinuity would lay the foundation for behaving in a certain way with a lexicographical preference order. For example, in its most extreme form, environmental quality would not figure as a driver of decisions in case there is a sufficient level of environmental quality around. However, once a threshold is passed, attention is diverted to the evolution of the environment and environmental quality starts to figure in the decision-taking process.

\section{The model}

In this model we consider an overlapping generations economy with an indeterminate horizon $t=0,1,2 \ldots$. We abstract from uncertainty by assuming perfect foresight and also from incomplete markets by taking them as perfectly competitive. Population is assumed to be constant and each generation consists of a single representative individual. A generation lives for two periods, young and old. The young generations have an inelastic labor supply and either save or invest in abatement. The old generations obtain utility from consuming their savings and from the state of the environment. So far, this model follows John and Pecchenino (1994). Our extension, as motived in the previous section, is to introduce a threshold in environmental quality. If environmental quality is above that threshold, then agents care relatively little about the environment, while if it is below that threshold, then preferences get directed towards environmental quality. Our intention is to study the impact of this threshold on the equilibria of the model and the dynamics.

\subsection{The environment}

We assume that the evolution of environmental quality $E_{t}$ is described by

$$
E_{t+1}=E_{t}-\beta c_{t}+\gamma A_{t}
$$

where $\beta>0$ is a parameters of consumption externality and represents the rate at which a unit of consumption $c_{t}$ pollutes the environment, while $\gamma>0$ represents the strength 
at which the abatement effort $A_{t}$ improves environmental quality. Hence, the stock of tomorrow's environment is depending on today's stock of the environment and is being reduced by consumption and improved through abatement. ${ }^{7}$ One deviation in this law of motion for environment from the original model of John and Pecchenino (1994) is that we assume away a natural regeneration rate. As a consequence, our model is applicable to cases where the environment cannot improve its natural state itself. This is, for example, a reasonable assumption for shallow lakes or irreversibility like soil erosion or climate change.

We also assume that $E_{t+i}=0, \forall i \geq 1$, if $E_{t}-\beta c_{t}+\gamma A_{t} \leq 0$. Consequently, once environmental quality has degenerated to its lowest level, then it cannot be improved again. We call $E=0$ the 'Point of Destruction'. Basically, we assume that life on earth would be impossible when environmental quality reaches the zero lower bound. In section 4 we discuss under what circumstances we may approach the Point of Destruction.

\subsection{The agents}

Agents receive a wage $w_{t}$ which they either save $s_{t}$ or use for abatement $A_{t}$. They earn an interest $R_{t+1}>1$ on their savings $s_{t}$ and consume from this $c_{t+1}$. Thus their budget constraints are $w_{t}=s_{t}+A_{t}$, and $c_{t+1}=R_{t+1} s_{t}$. Agents derive utility over consumption and environmental quality in period $t+1$ only. However, as motivated in the previous section, their marginal utility of environmental quality when old depends on the level of

\footnotetext{
${ }^{7}$ We could have also followed Jouvet et al. (2005) by assuming that the evolution of environmental quality is described by

$$
E_{t+1}=\tilde{E}+m\left(E_{t}-\tilde{E}\right)-\beta c_{t}+\gamma A_{t},
$$

where $m \in(0,1)$ is the speed at which the environment returns to its natural state $\tilde{E}>0$. This equation for environmental quality would have the advantage that it allows to keep track of the position of the environment relative to its natural state, to which it would always return in the absence of human intervention. It effectively is a linearized version of the Verhulst equation. However, apart from being able to describe the position of the environment relative to its natural state, this formulation would add little substance to our framework but complicate the theoretical analysis substantially. We will discuss one implication of this law of motion in section 4.
} 
environmental quality when young. In other words, we endogenize the semi-elasticity of utility based on a step function. Specifically, it depends on the level of environmental quality relative to the threshold that changes their preferences. For simplicity we assume that utility is logarithmic and takes the form

$$
U\left(c_{t+1}, E_{t+1}, \theta_{t}\right)=\ln c_{t+1}+\theta_{t} \ln E_{t+1},
$$

where $\theta_{t} \geq 0$ measures the relative preference of the generations for the environment over consumption. Thus, $\theta_{t}$ is also the semi-elasticity of environmental quality in utility. We now define the effect of the threshold.

Assumption 1 Assume that there exists a threshold, defined as $\widehat{E}>0$, then $\forall E_{t}<\widehat{E}$ we have $\theta_{t}=\theta_{H}$. Furthermore, $\forall E_{t} \geq \widehat{E}$ we have $\theta_{t}=\theta_{L}$. We assume that $\theta_{H}>\theta_{L}>0$.

Consequently, $\widehat{E}$ is the threshold level of environmental quality that induces a change in the generations' preferences. If a young generation is faced with a level of environmental quality which is below that threshold, then its preferences will be more strongly directed towards environmental quality than that of a young generation that faces a level of the environment which is above that threshold. ${ }^{8}$ Various motivations underlying the existence of this threshold have been given in the previous section.

\subsection{Solving the model}

The agents' problem is to maximize the utility function subject to the two budget constraints and environmental quality. Our approach for solving this model is to derive the first-order condition, show optimality, solve for the resulting equilibria and discuss convergence. Comparative statics complete the analysis.

Maximizing equation (3) subject to the budget constraints and equation (2) leads to

$$
R_{t+1} E_{t+1}=\gamma \theta_{t} c_{t+1},
$$

\footnotetext{
${ }^{8}$ In consequence, the correct terminology for our time-varying preferences is that preferences are predetermined.
} 
where, depending on the previous state of environmental quality, the endogenous evolution of the preferences follows: $\theta_{t}=\theta_{L}$ if $E_{t} \leq \widehat{E}$ and $\theta_{t}=\theta_{H}$ if $E_{t}<\widehat{E}$.

This first-order condition in equation (4) implies that the marginal benefit of saving one more unit wealth, and thus enjoying a larger consumption level when old, should be equal to the marginal cost of facing a lower environmental quality. Slightly re-writing equation (4) gives the condition $E_{t}-\beta c_{t}+\gamma A_{t}=\theta_{t} \gamma\left(w_{t}-A_{t}\right)$. We can see that increasing abatement by one unit improves environmental quality by $\gamma$ units. The cost of this is given by the reductions in savings which are valued by $\theta_{t} \gamma$. The main difference here to the framework of John and Pecchenino is the existence of a threshold that leads to a timevarying $\theta$ which induces discrete changes to the optimal solution in case the threshold is crossed.

The second-order condition is

$$
-\frac{1}{s_{t}}-\gamma^{2} \frac{\theta_{t}}{E_{t+1}^{2}}<0 .
$$

As a consequence, we know that our maximization problem fulfills the sufficient condition for optimality.

The assumptions and conditions above lead to the temporal equilibrium. It is defined as follows.

Definition 1 The temporal equilibrium consists of the allocations $\left\{w_{t}, R_{t}, k_{t}, c_{t}, s_{t}, A_{t}, E_{t}\right\}$, where at $t=0,1,2, \ldots$ firms maximize profits; labor and good markets clear; agents maximize (3) subject to their budget constraints $w_{t}=s_{t}+A_{t}$ and $c_{t+1}=R_{t+1} s_{t}$ and equation (2); net profits are distributed to the capital owners such that $R_{t}=f^{\prime}\left(k_{t}\right)$.

We now assume that the savings at time $t$ of the young generation are used for production and thus become the capital stock at $t+1, s_{t}=k_{t+1}$. This leads to $c_{t+1}=R_{t+1} k_{t+1}$ and $w_{t}-k_{t+1}=A_{t}$. Following the literature we suggest the standard Cobb-Douglas function as an explicit functional form for the production function, implying that $f(k)=k^{\alpha}$, with $\alpha \in(0,1)$. Like de La Croix and Michel (2002) we assume that capital fully depreciates during the course of one generation. 
As a consequence, equation (4) simplifies to

$$
E_{t}=\gamma \theta_{t-1} k_{t}
$$

Therefore, environmental quality will be proportional to the capital stock, where the factor of proportionality is given by $\gamma \theta_{t}$. The factor of proportionality depends on whether $E_{t-1}$ is smaller or larger than the threshold $\widehat{E}$. If $E_{t-1}<\widehat{E}$, then the factor of proportionality is $\theta_{H} \gamma$, while for $E_{t-1}>\widehat{E}$, then the factor of proportionality is $\theta_{L} \gamma$. Thus, for a low level of environmental quality when young, the agent will have preferences that are more strongly directed towards environmental quality and a larger share of wages will be spent on abatement. As a consequence, the ratio of environmental quality to capital will be larger for those old generations that have started off with a relatively low level of environmental quality.

Combining and solving the above equations gives the dynamic system that gives the intertemporal equilibrium, which can be fully described by

$$
\begin{aligned}
E_{t+1} & =\frac{\theta_{t}}{1+\theta_{t}}\left[E_{t}+((1-\alpha) \gamma-\alpha \beta)\left(\frac{E_{t}}{\gamma \theta_{t-1}}\right)^{\alpha}\right] \\
& \equiv H\left(E_{t}, \theta_{t-1}, \theta_{t}\right),
\end{aligned}
$$

and where we have the threshold condition $\theta_{t}=\theta_{L}$ if $E_{t} \leq \widehat{E}$ and $\theta_{t}=\theta_{H}$ if $E_{t}<\widehat{E}$.

This allows us now to define the intertemporal equilibrium.

Definition 2 Given $k_{0}$ and $E_{0}$, an intertemporal equilibrium is a temporal equilibrium that, for all $t \geq 0$, satisfies the capital accumulation condition $s_{t}=k_{t+1}$ as well as equation (7).

Our focus is on studying and describing this intertemporal equilibrium. This will be complicated insofar as function $H\left(E_{t}, \theta_{t-1}, \theta_{t}\right)$ is subject to discrete changes whenever environmental quality crosses the threshold $\widehat{E}$. Function $H$ is a strictly increasing concave function in $E_{t}$ if $\kappa \equiv \frac{(1-\alpha) \gamma-\alpha \beta}{\gamma^{\alpha}}>0$.

In consequence, the evolution of $E_{t}$ depends on both the returns to abatement relative to the polluting effect of consumption, the share of wealth directed towards abatement, as 
well as the level of $E_{t}$ relative to the threshold. If $\kappa>0$, then the effective share of wealth spent on abatement $((1-\alpha) \gamma)$ offsets the emissions created from allocating a share of wealth to consumption $(\alpha \beta)$. We call this the 'strong abatement' case. Mathematically we get that $H\left(E_{t}, \theta_{t-1}, \theta_{t}\right)$ is a monotonically increasing function in $E_{t}$ if $\kappa>0$. The intuition is that in the case of strong abatement, increases to capital have a positive net effect on environmental quality, as the abatement effort always offsets the emissions. Thus, increases in wealth also lead to improvements in the environment.

In the case of 'too dirty consumption', i.e. when $\kappa<0$, the abatement efforts are too

small to be able to offset the emissions. Consequently, the term $((1-\alpha) \gamma-\alpha \beta)\left(\frac{E_{t}}{\theta_{t-1 \gamma}}\right)^{\alpha}$ has a negative impact on future environmental quality. Since it describes the net effect of abatement versus emissions, then this implies that the current and future environmental stocks are essentially related through a negative feedback loop. We will treat the 'too dirty consumption' case, i.e. $\kappa<0$, more fully in section $4 .{ }^{9}$ For $\kappa<0$, we have that $H\left(E_{t}, \theta_{t-1}, \theta_{t}\right)<E_{t}$. Thus, if $\kappa<0$ then $E_{t+1}<E_{t}$ and we will converge to the Point of Destruction.

For now, we shall only focus on the more involved case of $\kappa>0$.

Assumption 2 We assume that $\kappa \equiv \frac{(1-\alpha) \gamma-\alpha \beta}{\gamma^{\alpha}}>0$.

Thus, henceforth, we deal with the case where the effective share of income dedicated to abatement outweighs the effective share of income that is polluting.

\subsection{Steady state and dynamics}

For the analysis of the steady state and the dynamics it is sufficient to focus on equation (7). There will be essentially three different regimes. We pick up the two simple regimes first. Assuming that environmental quality over time does not change and simplifying leads to

$$
E_{i}=\theta_{i} \kappa^{\frac{1}{1-\alpha}}
$$

\footnotetext{
${ }^{9}$ For simplicity we neglect the case of $\kappa=0$, since it is a degenerate parameter combination.
} 
for $i=L, H$, where $\theta_{i}=\theta_{L}$ if $E_{L}>\widehat{E}$, while $\theta_{i}=\theta_{H}$ if $E_{H}<\widehat{E}$. Since $\theta_{L}<\theta_{H}$, we also know that $E_{L}<E_{H}$. Thus we can distinguish the first two regimes.

Case I (low threshold): $\widehat{E}<E_{L}<E_{H}$. By intuition, we would expect the following. Let us assume that only very low levels of environmental quality induce a change in the preferences. A society fixated on consumption or a country with a currently low level of environmental awareness (c.f. Prieur and Bréchet 2013) could fit this profile. In this case one could argue that a society like this would not be willing to invest a large share of income in the environment unless environmental quality becomes painfully low. In other words, this would, for example, be the case for a society that is strongly detached from the environment and only includes the externality environment in its decision-taking progress when survival is at stake. In the case where environmental quality is below this threshold where the society's concern for the environment becomes important, then this society will invest strongly in environmental quality. However, in the moment that the society feels out of danger, i.e. the preference threshold is crossed, then the preferences will return to a point where little importance is given to the environment. As long as some abatement effort is undertaken, meaning as long as that society wants to avoid the Point of Destruction, environmental quality will converge to a small but positive level. We summarize this intuition in the following proposition and prove it analytically.

Proposition 1 Suppose $\widehat{E}<\theta_{L} \kappa^{\frac{1}{1-\alpha}}<\theta_{H} \kappa^{\frac{1}{1-\alpha}}$, then there exists a unique asymptotic stable positive steady state, which is given by $E_{L}=\theta_{L} \kappa^{\frac{1}{1-\alpha}}$.

Proof 1 In this case, either there exits some $t_{0}>0$, such that, for all $t>t_{0}, E_{t}<\widehat{E}$, or for $t>t_{0}, E_{t}>\widehat{E}$.

If $E_{t}<\widehat{E}$, we must have $\theta_{t}=\theta_{H}$ by assumption. Therefore, the dynamic equation is

$$
E_{t+1}=\frac{\theta_{H}}{1+\theta_{H}}\left(E_{t}+\frac{\kappa}{\theta_{H}^{\alpha}} E_{t}^{\alpha}\right) .
$$

Thus, the steady state, if it were to exist, would have to satisfy

$$
\kappa^{\frac{1}{1-\alpha}} \theta_{H}=E_{H} .
$$


However, this leads to a contradiction, since by assumption $\widehat{E}<E_{L}$ and $E_{L}<E_{H}$. Therefore, there exists a $t_{0}>0$, such that for $t>t_{0}, E_{t}>\widehat{E}$ and hence, $\theta_{t}=\theta_{L}$. From the dynamic equation

$$
E_{t+1}=\frac{\theta_{L}}{1+\theta_{L}}\left(E_{t}+\frac{\kappa}{\theta_{L}^{\alpha}} E_{t}^{\alpha}\right),
$$

we thus obtain the unique positive steady state given by $E_{L}=\theta_{L} \kappa^{\frac{1}{1-\alpha}}$.

Convergence to $E_{L}$ can be proven as follows. From the dynamic equation $E_{t+1}=$ $\frac{\theta_{L}}{1+\theta_{L}}\left(E_{t}+\frac{\kappa}{\theta_{L}^{\alpha}} E_{t}^{\alpha}\right)$, we have that $E_{t+1}<E_{t}$ if $E_{t}>\theta_{L} \kappa^{1-\alpha}$. Since $E_{L}=\theta_{L} \kappa^{1-\alpha}$ then this implies that $E_{t+1}<E_{t}$ for $E_{t}>E_{L}$. A similar argument implies that $E_{t+1}>E_{t}$ for $E_{t}<E_{L}$. We thus proved convergence to $E_{L}$ from above and below.

We now turn to our second case.

Case II (high threshold): $E_{L}<E_{H}<\widehat{E}$. This condition can be expected to be fulfilled in a society in which the environment plays an important part of people's lives. For example, a society that has strong moral norms directed towards the global good, or one that has a more holistic approach to life. This would be the case of many earlier societies which were trying to live with the environment instead of purely benefiting from it. In this case the society will keep the high preference towards the environment for normal (i.e. those for which $E_{t}<\widehat{E}$ ) levels of the environment. Thus, it can be expected that a society with preferences that are more strongly directed to the environment will also prefer to live in a world with a higher level of environmental quality. ${ }^{10}$ We summarize this case in the following proposition, which can apply a similar argument as case I.

Proposition 2 Suppose $\theta_{L} \kappa^{\frac{1}{1-\alpha}}<\theta_{H} \kappa^{\frac{1}{1-\alpha}}<\widehat{E}$, then the dynamic system converges to a unique asymptotic stable positive steady state, given by $E_{H}=\theta_{H} \kappa^{\frac{1}{1-\alpha}}$.

\section{Proof 2 The proof is similar to Case I.}

\footnotetext{
${ }^{10}$ In the extreme case of $\widehat{E}=\infty$, the society will never change its preferences and always consider the environment as relatively important for utility.
} 
These two cases, the low threshold and the high threshold one, essentially lead to the a unique globally asymptotically stable equilibrium as it is the case with the original John and Pecchenino model without changing preferences. However, a crucial role is still played by the position of the threshold relative to the potential steady states. We clearly showed that societies that generally live in a benign state of environmental neglect will always pick up the steady state with the bad environmental quality, even if for some time their preferences change and place a more important emphasis on the environment. Therefore, in societies that are characterized by a sort of short spurts of environmental support, or those that act only due to pressing needs, we will also observe relatively low long-term levels of environmental quality. In contrast, in societies that are built upon a stronger connection towards the environment we will see that environmental quality will be allowed to grow to a relatively high long-run level, even if the society were to change its preferences to one with a lower emphasis on environmental quality if the state of the environment is very high.

We now deal with the last, somewhat more complicated case, where the preference threshold has an intermediate level. This leads to less clear-cut results.

Case III (intermediate threshold): $E_{L}<\widehat{E}<E_{H}$.

In this case we can quite easily show that neither $E_{H}$ nor $E_{L}$ will turn out to be a steady state. From equation (7), if $\theta_{t-1}=\theta_{t}=\theta_{L}$ ( which means $E_{t} \geq \widehat{E}$, for any $t$ ) then we obtain $E_{L}=\theta_{L} \kappa^{\frac{1}{1-\alpha}}$. Hence, $E_{L} \geq \widehat{E}$. Similarly, if $\theta_{t-1}=\theta_{t}=\theta_{H}$ ( which means $E_{t} \leq \widehat{E}$, for any $t$ ), then $E_{H}=\theta_{H} \kappa^{\frac{1}{1-\alpha}}$. Therefore, $E_{H} \leq \widehat{E} \leq E_{L}$. However, in the intermediate threshold case we have that $E_{L}<\widehat{E}<E_{H}$, which is equivalent to $\theta_{L} \kappa^{\frac{1}{1-\alpha}}>\widehat{E}>\theta_{H} \kappa^{\frac{1}{1-\alpha}}$. The above is true if and only if $\theta_{L}>\theta_{H}$, which however contradicts our Assumption 1 that $\theta_{L}<\theta_{H}$. Thus, we conclude that $E_{L}$ and $E_{H}$ cannot be steady states in the intermediate threshold case.

Intuitively, what we would expect now is the following. For a high level of environmental quality, such that $E_{t}>\widehat{E}$, a society will place little relative importance on the environment as environmental quality is, in its eyes, sufficiently high. Thus, capital accumulation will be increased in order to have a larger consumption when old, which directly 
leads to a lower level of abatement and consequently a reduction in environmental quality. This reduction will continue until environmental quality passes the threshold at which society suddenly notices that the environment is strongly degraded and starts to place more emphasis on abatement and the recovery of environmental quality. Given the assumption of $E_{L}<\widehat{E}<E_{H}$, the society, due to its preferences being more strongly directed to the environment $^{11}$, now targets a high level of environmental quality (given by $E_{H}$ ). However, having reached a sufficiently high level of environmental quality, and thereby having passed the threshold, the society now feels that it had placed too much relative emphasis on the environment and returns to its previous pollution-intensive behavior.

Following this logic implies that we should expect no convergence to a single point, but society will be caught in an endless feedback loop where preferences that are more strongly directed towards the environment lead to a higher environmental quality. This eventually leads to a crossing of the threshold, which induces lower relative preferences directed towards the environment, which in return leads to a reduction in environmental quality again. We now present the analytical underpinnings of this intuitive description.

Proposition 3 Suppose that $\theta_{L} \kappa^{\frac{1}{1-\alpha}}<\widehat{E}<\theta_{H} \kappa^{\frac{1}{1-\alpha}}$, then for any $p \in \mathbb{N}$ and $p \geq 2$, there exists a p-cycle for the dynamic equation (7) and the threshold conditions $\theta_{t}=\theta_{L}$ if $E_{t} \leq \widehat{E}$ and $\theta_{t}=\theta_{H}$ if $E_{t}<\widehat{E}$.

Proof 3 The logic of the proof is the following. We first provide the argument and condition under which a 2-cycle may occur, then the conditions under which a 3-cycle may occur, and then conclude on the existence of a p-cycle.

Suppose that at $t-1, E_{t-1}>\widehat{E}$, then we consequently have $\theta_{t-1}=\theta_{L}$; while at period $t, E_{t}<\widehat{E}$, and hence society will pick up $\theta_{t}=\theta_{H}$. That yields $E_{t+1}=H\left(E_{t}, \theta_{L}, \theta_{H}\right)$. A 2-cycle would imply that $E_{t+1}=E_{t-1}>\widehat{E}$ and, similarly, we would also have $E_{t+2}=$ $H\left(E_{t+1}, \theta_{H}, \theta_{L}\right)=E_{t}<\widehat{E}$. We now denote the two levels of environmental quality where these conditions are satisfied as $E_{L H}=H\left(E_{H L}, \theta_{L}, \theta_{H}\right)$ and $E_{H L}=H\left(E_{L H}, \theta_{H}, \theta_{L}\right)$.

\footnotetext{
${ }^{11}$ This is, within this model, meant in the sense of a higher semi-elasticity of environmental quality in utility.
} 
These two equilibrium points can then be written as

$$
\begin{aligned}
& E_{L H}=\frac{\theta_{H}}{1+\theta_{H}}\left(E_{H L}+\kappa\left(\frac{E_{H L}}{\theta_{L}}\right)^{\alpha}\right), \\
& E_{H L}=\frac{\theta_{L}}{1+\theta_{L}}\left(E_{L H}+\kappa\left(\frac{E_{L H}}{\theta_{H}}\right)^{\alpha}\right) .
\end{aligned}
$$

We claim that this system of equations gives one and only one positive solution. Indeed, substituting $\frac{E_{L H}}{\theta_{H}}=\frac{1}{1+\theta_{H}}\left(E_{H L}+\kappa\left(\frac{E_{H L}}{\theta_{L}}\right)^{\alpha}\right)$ into equation (10), it follows

$$
E_{H L}=\frac{\theta_{L}}{1+\theta_{L}}\left[\frac{\theta_{H}}{1+\theta_{H}}\left(E_{H L}+\kappa\left(\frac{E_{H L}}{\theta_{L}}\right)^{\alpha}\right)+\frac{\kappa}{\left(1+\theta_{H}\right)^{\alpha}}\left(E_{H L}+\kappa\left(\frac{E_{H L}}{\theta_{L}}\right)^{\alpha}\right)^{\alpha}\right] .
$$

Or equivalently, we can rewrite the above as

$$
\begin{gathered}
E_{H L}\left(1-\frac{\theta_{L}}{1+\theta_{L}} \frac{\theta_{H}}{1+\theta_{H}}\right) \\
=\frac{\theta_{L}}{1+\theta_{L}}\left[\frac{\theta_{H}}{1+\theta_{H}} \kappa\left(\frac{E_{H L}}{\theta_{L}}\right)^{\alpha}+\frac{\kappa}{\left(1+\theta_{H}\right)^{\alpha}}\left(E_{H L}+\kappa\left(\frac{E_{H L}}{\theta_{L}}\right)^{\alpha}\right)^{\alpha}\right],
\end{gathered}
$$

in which the left hand side is a straight line in term of $E_{H L}$ starting from 0 and goes to $\infty$, while the right hand side is a strictly increasing concave function, starting at zero. Thus, we can prove that there is one and only one positive solution $E_{H L}$ which satisfies the above equation. The same obviously holds for $E_{L H}$.

As a byproduct, and based on the above notation, we have the following.

Corollary 1 Suppose that $\theta_{L} \kappa^{\frac{1}{1-\alpha}}<\widehat{E}<\theta_{H} \kappa^{\frac{1}{1-\alpha}}$, then it follows that

$$
E_{H L}<\widehat{E}<E_{L H}
$$

Consequently, this pair of equations forms a stable 2-cycle. Following a similar logic as above, we can prove the existence of a 3-cycle. For this we have to distinguish two cases, which depend on the relative position of the threshold. 
In $\underline{\text { Case I }}$ we assume that at time $t-1$ and $t$, we have $E_{t-1}>\widehat{E}$ and $E_{t}>\widehat{E}$, while at $t+1$, we have $E_{t+1}<\widehat{E}$. Moreover, the following dynamics hold

$$
\left\{\begin{array}{l}
E_{t+2}=G\left(E_{t+1}, \theta_{t}, \theta_{t+1}\right)=G\left(E_{t+1}, \theta_{L}, \theta_{H}\right)=E_{t-1} \\
E_{t+1}=G\left(E_{t}, \theta_{t-1}, \theta_{t}\right)=G\left(E_{t}, \theta_{L}, \theta_{L}\right)=E_{t-2} \\
E_{t}=G\left(E_{t-1}, \theta_{t-2}, \theta_{t-1}\right)=G\left(E_{t-1}, \theta_{H}, \theta_{L}\right)=E_{t-3}
\end{array}\right.
$$

Thus, the 3-cycle is defined by

$$
E_{t+2}=E_{t-1}, E_{t+1}=E_{t-2}, E_{t}=E_{t-3},
$$

and in short, we use the following notations:

$$
E_{H L H}=G\left(E_{L L L}, \theta_{L}, \theta_{H}\right), E_{L L L}=G\left(E_{L H L}, \theta_{L}, \theta_{L}\right), E_{L H L}=G\left(E_{H L H}, \theta_{H}, \theta_{L}\right) .
$$

The 3-cycle is then the solution of the following system

$$
\left\{\begin{array}{l}
E_{H L H}=\frac{\theta_{H}}{1+\theta_{H}}\left(E_{L L L}+\kappa \frac{E_{L L L}^{\alpha}}{\theta_{L}^{\alpha}}\right), \\
E_{L L L}=\frac{\theta_{L}}{1+\theta_{L}}\left(E_{L H L}+\kappa \frac{E_{L H L}^{\alpha}}{\theta_{L}^{\alpha}}\right), \\
E_{L H L}=\frac{\theta_{L}}{1+\theta_{L}}\left(E_{H L H}+\kappa \frac{E_{H L H}^{\alpha}}{\theta_{H}^{\alpha}}\right) .
\end{array}\right.
$$

The unique positive solution of this system, which can be demonstrated in the same way as in the case of the 2-cycle, generates a 3-cycle.

Using a similar argument we have the second case.

In Case II we have that at time $t-1$ and $t$, there is $E_{t-1}<\widehat{E}$ and $E_{t}<\widehat{E}$, but at $t+1$, it yields $E_{t+1}>\widehat{E}$. Moreover, the following dynamics hold

$$
\left\{\begin{array}{l}
E_{t+2}=G\left(E_{t+1}, \theta_{t}, \theta_{t+1}\right)=G\left(E_{t+1}, \theta_{H}, \theta_{L}\right)=E_{t-1} \\
E_{t+1}=G\left(E_{t}, \theta_{t-1}, \theta_{t}\right)=G\left(E_{t}, \theta_{H}, \theta_{H}\right)=E_{t-2} \\
E_{t}=G\left(E_{t-1}, \theta_{t-2}, \theta_{t-1}\right)=G\left(E_{t-1}, \theta_{L}, \theta_{H}\right)=E_{t-3}
\end{array}\right.
$$

and thus the 3-cycle is defined similarly to above as

$$
E_{t+2}=E_{t-1}, E_{t+1}=E_{t-2}, E_{t}=E_{t-3},
$$


Thus, using a similar notation

$$
E_{L H L}=G\left(E_{H H H}, \theta_{H}, \theta_{L}\right), E_{H H H}=G\left(E_{H L H}, \theta_{H}, \theta_{H}\right), E_{H L H}=G\left(E_{L H L}, \theta_{L}, \theta_{H}\right),
$$

by the same arguments we obtain a 3-cycle. In a similar vain we can derive a p-cycle, for any $p \geq 2$. That completes the proof.

In the following, we study the domain of the potential cycles. This result is summarized in the following proposition.

Proposition 4 Suppose that $\theta_{L} \kappa^{\frac{1}{1-\alpha}}<\widehat{E}<\theta_{H} \kappa^{\frac{1}{1-\alpha}}$, then any $E_{t}$ converges to a $p$-cycle and stays between $E_{L}$ and $E_{H}$.

Proof 4 See Appendix.

It is, unfortunately, not possible to derive which frequency of cycle we should expect under what parameter conditions. Indeed, given the fact that there are infinitely many $p$-cycles, then, as shown above, each $E_{t} \in\left(E_{L}, E_{H}\right)$ is linked to one cycle. Consequently, for $E_{L}<\hat{E}<E_{H}$, if $E_{t} \in\left(E_{L}, E_{H}\right)$, then we are already on a p-cycle.

Thus, it is impossible to know whether preferences change every generation, which would be the case in a 2-cycle, or whether it takes several generations for the preferences to change, which would be the case of a cycle of higher order.

This result is the crucial difference to John and Pecchenino, and the role of the threshold becomes the most obvious. Consequently, what is important to take away is the fact that this threshold in preferences can induce cycles if the threshold is neither too high nor too low. Knowing the order of the cycles would be useful for empirical testing. Apart from that, however, the result that cycles can occur - independent of knowing their order - has implications for intergenerational equity.

In terms of intergenerational equity, the results that we obtain here follow the same argument as outlined in Schumacher and Zou (2008). Basically, these cycles may induce a violation of standard criteria of intergenerational equity, e.g. non-decreasing utility. And, 
in contrast to the common argument that climate change or environmental destruction may lead to a violation of intergenerational equity in the distant future, we conclude that violations are possible already in the near future. As a consequence, this gives rise to policy interventions that help in avoiding the violations in intergenerational equity, which we discuss in section 5 after having gone over some points related to the robustness of the model.

\section{Robustness}

In this section we show under which circumstances this economy would converge to the Point of Destruction $\left(E_{t} \rightarrow 0\right)$. Also, we discuss the role of a natural regeneration rate as well as the implication of our assumption $\kappa>0$.

In section 3.1 we defined the 'Point of Destruction' as the level of the environment where environmental quality is equal to zero. Intuitively, it is the level at which disaster occurs and life on earth becomes unsustainable. Mathematically we defined this level to be $E_{t+i}=0, \forall i \geq 1$, if $E_{t}-\beta c_{t}+\gamma A_{t} \leq 0$. From the previous analysis we know that the only way in which we may have $E_{t}=0$ as a steady state solution is if the marginal utility of environmental quality becomes negligible small for agents' decisions. This occurs only for $\theta_{L}=0$. However, this only insures that the Point of Destruction may actually be a potential equilibrium point. The point is that, for any $\hat{E}>0$, preferences change and the new preference parameter becomes $\theta_{H}$. Consequently, the only way in which environmental quality may actually approach zero requires a combination of conditions. From the maximization problem we can easily show that, for $\theta_{L}=0, A_{t}=0$. Thus, $E_{t+1}=E_{t}-\beta w_{t}$. Consequently, we require that $\hat{E}$ is sufficiently low, meaning if there $\exists i>0$, such that $E_{i}-\beta w_{i} \leq 0$, then we require $\hat{E} \in\left(0, E_{i}\right)$. As a result, convergence to the Point of Destruction is really a result of an extreme case that furthermore requires a sufficient myopia.

We can generalize this result. For example, we may assume a natural regeneration rate along the lines of Jouvet et al. (2005) and suggest that the evolution of environmental 
quality is described by

$$
E_{t+1}=\tilde{E}+m\left(E_{t}-\tilde{E}\right)-\beta c_{t}+\gamma A_{t}
$$

where $m \in(0,1)$ is the speed at which the environment returns to its natural state $\tilde{E}>0$.

In this case we require a third condition for environmental quality to converge to the Point of Destruction, namely that the natural regeneration $m E_{t}$ must be smaller than the total amount of pollution. Within our notation this turns out to be $m E_{t}<(1-\alpha) \beta k_{t}^{\alpha}$.

There is another condition that would lead environmental quality to converge to the Point of Destruction. It is given by $\kappa<0$. In this case, the effective share of wages spent on abatement is smaller than the total amount of pollution that arises from the consumption activities. In this case, equation (7) predicts that environmental quality will necessarily decrease over time and converge to the Point of Destruction. Thus, the model would essentially predict a destruction of the environment, no matter where the threshold is located and how strongly preferences are directed towards the environment. This, obviously, is a weakness of modeling without a natural regeneration rate and with log-utility. However, this weakness is only relevant if $\kappa<0$. Also, the assumption of a sufficiently high regeneration rate $m>0$ would then allow for a positive steady state in environmental quality. It would, nevertheless, draw away attention from the importance of the threshold, which clearly comes as an important driver of dynamics within our current model setting.

\section{$5 \quad$ Policy making}

For this policy analysis we focus on the role of the threshold, since this is the main contribution of our work. We start of by studying inhowfar the threshold affects society's indirect utility. We then present a method with which policies can be compared even though preferences are endogenous. Then we analyze a potential way in which a policy maker can shift society to the steady state with higher indirect utility. We distinguish between costless policies and those that are costly and compare those that are financed 
via an income tax with those that are financed through a lump-sum tax.

\subsection{Costless policies}

We want to know which society is better off in terms of indirect utility, namely the one with a high threshold of environmental quality or the one with a lower one. We can solve for this as follows. From the two dynamic equations (4) and (6), it is easy to see that the two steady states of capital and consumptions are given by, for $i=L, H$,

$$
k_{i}=\frac{E_{i}}{\gamma \theta_{i}}=\frac{\kappa^{\frac{1}{1-\alpha}}}{\gamma}, \quad c_{i}=\frac{R_{i} E_{i}}{\gamma \theta_{i}}=\alpha\left(\frac{E_{i}}{\gamma \theta_{i}}\right)^{\alpha}=\alpha k_{i}^{\alpha}=\frac{\alpha \kappa^{\frac{\alpha}{1-\alpha}}}{\gamma^{\alpha}} .
$$

Indirect utility (3), for $i=L, H$, at steady state is then given by

$$
U_{i}=\ln \left(c_{i}\right)+\theta_{i} \ln \left(E_{i}\right)=\ln \left(\frac{\alpha \kappa^{\frac{\alpha}{1-\alpha}}}{\gamma^{\alpha}}\right)+\theta_{i} \ln \left(\theta_{i} \kappa^{\frac{1}{1-\alpha}}\right),
$$

which is a strictly increasing convex function of $\theta_{i}$.

Result 1 The position of the threshold determines indirect utility since it determines the level of environmental quality at steady state.

In terms of policy making, Result 1 implies that if the threshold exceeds $E_{H}$, then this leads to the highest indirect utility at steady state. In contrast, for the threshold below $E_{L}$, we conclude that indirect utility at steady state will be the lowest. ${ }^{12}$ This suggests that, if there are means of affecting this threshold, there could be a potential role for policy interventions. Let us assume a policy maker can freely choose $\hat{E}$. This would, for example be a reasonable assumption in case the threshold arises due to environmental standards that are based on health assessments. Then the question would be: Under what circumstances would a policy maker want to set $\hat{E}$ to $\hat{E}>E_{H}$ ? We know that indirect utility at steady state under $\hat{E} \geq E_{H}$ is higher than under $\hat{E}<E_{H}$. However, this is due to the discrete preference change whenever $E$ passes the threshold. Here

\footnotetext{
${ }^{12}$ This holds obviously only if we do not converge to the Point of Destruction.
} 
we enter the territory described by Marshak (1978), where public policy directly shapes preferences or tastes. Marshak questions this kind of policy intervention on the grounds that (p.386) "if one continues to believe that even in a world of changeable tastes the foundation for policy and prediction has to be a theory of individual rational choice, then one risks turning Economic Man into a complex monster of calculated schizophrenia, who chooses or manipulates future mutations of himself." Consequently, as many economists would argue, if preferences change, then an intrapersonal comparison may be meaningless and the evaluation of a policy impossible. Nevertheless, a policy evaluation is possible if all possible future selfs were to hold the same qualitative opinion on the policy. These future selfs are: agent $\mathrm{A}$, who has the new preferences and prefers the $\{c, E\}$ bundle obtained based on those new preferences; agent B, who holds the old preferences but would prefer the $\{c, E\}$ bundle obtained based on the new preferences. For a clear-cut case, let us assume that, initially, $\hat{E}<E_{L}$. The policy maker has now the possibility to raise $\hat{E}$ (e.g. by changing the environmental standard) free of charge. We argue here that this policy should be undertaken if $u\left(\theta_{L}, c\left(\theta_{H}\right), E\left(\theta_{H}\right)\right)>u\left(\theta_{L}, c\left(\theta_{L}\right), E\left(\theta_{L}\right)\right)$, and if $u\left(\theta_{H}, c\left(\theta_{H}\right), E\left(\theta_{H}\right)\right)>u\left(\theta_{H}, c\left(\theta_{L}\right), E\left(\theta_{L}\right)\right)$. In this case, all possible future selfs are better off and the policy is worthwhile to undertake. We now evaluate these cases in turn.

Agent A compares $u\left(\theta_{H}, c\left(\theta_{H}\right), E\left(\theta_{H}\right)\right)>u\left(\theta_{H}, c\left(\theta_{L}\right), E\left(\theta_{L}\right)\right)$. Substituting the respective optimal solutions at the steady state values gives

$$
\ln \left(\left(\frac{\alpha E_{H}}{\gamma \theta_{H}}\right)^{\alpha}\right)+\theta_{H} \ln E_{H}>\ln \left(\left(\frac{\alpha E_{L}}{\gamma \theta_{L}}\right)^{\alpha}\right)+\theta_{H} \ln E_{L} .
$$

Simplifying gives

$$
E_{H}>E_{L}
$$

This holds by assumption. Thus, agent A is better off if the policy is undertaken.

Agent B evaluates $u\left(\theta_{L}, c\left(\theta_{H}\right), E\left(\theta_{H}\right)\right)>u\left(\theta_{L}, c\left(\theta_{L}\right), E\left(\theta_{L}\right)\right)$. This gives rise to

$$
\ln \left(\left(\frac{\alpha E_{H}}{\gamma \theta_{H}}\right)^{\alpha}\right)+\theta_{L} \ln E_{H}>\ln \left(\left(\frac{\alpha E_{L}}{\gamma \theta_{L}}\right)^{\alpha}\right)+\theta_{L} \ln E_{L} .
$$

Substituting leads again to the condition $E_{H}>E_{L}$. Consequently, both agents prefer that the policy is undertaken. In this case, one can argue that there are reasonable grounds 
for policy intervention, and that this policy intervention should be designed in such a way that the threshold takes the highest possible (or highest necessary) level in order to assure convergence to the high steady state. The same reasoning applies to the case where $\hat{E} \in\left(E_{L}, E_{H}\right)$. It is clear from the results in the previous section that in this case indirect utility cycles between $U_{H}$ and $U_{L}$, and as a result it is still worthwhile for the policy maker to set $\hat{E} \geq E_{H}$.

\subsection{Income tax}

The previous policy result rests on the assumption that it is costless to change threshold $\hat{E}$. While one could assume this to be true for a policy that sets health standards or alike, it is unlikely for thresholds that arise due to e.g. cultural dynamics. In this case, educational measures are helpful in driving public opinion. However, these tend to be costly (this is, e.g., the case in Prieur and Bréchet 2013). As a result, one could ask: What would be the maximum willingness to pay in order to set $\hat{E}$ to $\hat{E}>E_{H}$ ? Our comparison here will be again between the cases where $\hat{E}<E_{L}$ and where the policy maker wants to change $\hat{E}$ to $\hat{E}>E_{H}$.

Assume the policy maker raises an income tax $\tau$ which then pays for the change in the threshold. Since educational achievements are also subject to full depreciation during the course of a lifetime, this educational expenditure needs to be undertaken in every generation. Hence, the dynamic equation (7) changes to

$$
E_{t+1}=\frac{\theta_{t}}{1+\theta_{t}}\left[E_{t}+((1-\alpha) \gamma(1-\tau)-\alpha \beta)\left(\frac{E_{t}}{\gamma \theta_{t-1}}\right)^{\alpha}\right] \text {. }
$$

The high steady state will then be given by $E_{H}(\tau)=\theta_{H} \kappa(\tau)^{\frac{1}{1-\alpha}}$, where $\kappa(\tau) \equiv \frac{(1-\alpha) \gamma(1-\tau)-\alpha \beta}{\gamma^{\alpha}}$. Consequently, indirect utility differences at steady state between $U_{L}$ and $U_{H}$ give the maximum willingness to pay to shift $\hat{E}$ above $E_{H}$, which is given by

$$
\Omega(\tau) \equiv \ln \left(\frac{\alpha \kappa(\tau)^{\frac{\alpha}{1-\alpha}}}{\gamma^{\alpha}}\right)+\theta_{H} \ln \left(\theta_{H} \kappa(\tau)^{\frac{1}{1-\alpha}}\right)-\ln \left(\frac{\alpha \kappa^{\frac{\alpha}{1-\alpha}}}{\gamma^{\alpha}}\right)-\theta_{L} \ln \left(\theta_{L} \kappa^{\frac{1}{1-\alpha}}\right) .
$$

The maximum feasible level of $\tau$ is given by $\bar{\tau}=\frac{\kappa}{(1-\alpha) \gamma^{1-\alpha}}$. Function $\Omega(\tau)$ has the following shape. For $\tau=0$, we have $\Omega(\tau)=\theta_{H} \ln \left(\theta_{H} \kappa^{\frac{1}{1-\alpha}}\right)-\theta_{L} \ln \left(\theta_{L} \kappa^{\frac{1}{1-\alpha}}\right)>0$, while $\Omega(\bar{\tau})=-\infty$. 
Then, the derivative of $\Omega$ with respect to $\tau$ is

$$
\frac{\partial \Omega(\tau)}{\partial \tau}=-\frac{\left(\theta_{H}+\alpha\right) \gamma}{(1-\alpha) \gamma(1-\tau)-\alpha \beta}=-\frac{\left(\theta_{H}+\alpha\right) \gamma^{1-\alpha}}{\kappa(\tau)}<0 .
$$

As a result, the maximum willingness to pay for shifting $\hat{E} \geq E_{H}$ is given by $\tau^{\text {max }}$, where $\tau^{\max }$ is defined by $\Omega\left(\tau^{\max }\right)=0$.

In the light of the motivational part, and with the specific mechanism of environmental laws and standards in mind, then we may conclude from this that it is better to tighten laws and require tougher standards, since this would increase the threshold $\hat{E}$ and consequently increase the likelihood of converging to the high steady state and high indirect utility.

If the policy that changes the threshold is costly and if this policy is paid via a percentage income tax, then raising the threshold has the consequence that it also lowers the steady state in environmental quality. This arises since a part of income that otherwise could go towards abatement is being used up by the government to finance the raising of the threshold. As a result, indirect utility at steady is lowered (with respect to the status quo $E_{H}$ ) the more the policy maker has to tax the agents.

\subsection{Lump-sum tax}

Instead of an income tax a policy maker could also introduce a lump-sum tax. In this case, the wage constraint is modified and now reads

$$
w_{t}-\tau_{t}=s_{t}+A_{t}
$$

where $\tau_{t} \geq 0$ is the lump-sum tax. We assume that the agent takes the lump-sum tax as given when choosing the optimal allocations, and the policy maker chooses the tax 
afterwards. ${ }^{13}$ As a result, this modifies the dynamic equation (7), which now becomes

$$
E_{t+1}=\frac{\theta_{t}}{1+\theta_{t}}\left[E_{t}+\kappa \frac{E_{t}^{\alpha}}{\theta_{t}^{\alpha-1}}-\gamma \tau_{t}\right]
$$

In this case we find the steady state characterized by equation

$$
E=\kappa \theta_{i}^{1-\alpha} E^{\alpha}-\gamma \theta_{i} \tau, \quad i=L, H
$$

We assume again that the policy maker only introduces the tax if this helps in raising the threshold such that the preference parameter $\theta_{H}$ applies and if indirect utility increases. As a result of this tax, we notice that the steady state level of environmental quality decreases, and so does next period's level of the environment. This happens since the tax works as a reduction to wages such that net wages available to finance both savings and abatement expenditure are lower.

From equation (18), we see that the left-hand side is a linear function of $E$ and the right hand side, $g(E ; \tau)=\kappa \theta_{H}^{1-\alpha} E^{\alpha}-\gamma \theta_{H} \tau$, is an increasing and concave function in $E$ while it decreases in $\tau$. If $\tau=0$, we revert to the previous case where the steady states depend on the position of the threshold relative to the steady states $E_{L}$ and $E_{H}$. If $\tau>0$, the right hand side $g(E ; \tau)$ decreases and there exists a $\bar{\tau}>0$, such that,

(a) if $0<\tau<\bar{\tau}$, there are two positive steady state: $E^{l}(\tau)$ and $E^{h}(\tau)$ with $E^{l}(\tau)<$ $E^{h}(\tau)<E_{H}$.

(b) at $\tau=\bar{\tau}$, there is one positive steady state $E^{w}(\bar{\tau})<E_{H}$, located at $E^{l}(\tau)<$ $E^{w}(\bar{\tau})<E^{h}(\tau)<E_{H}$.

(c) if $\tau>\bar{\tau}$, there is no steady state.

We can now demonstrate the following results.

Proposition 5 For $\tau \in(0, \bar{\tau})$, the steady state $E^{l}(\tau)$ is unstable while $E^{h}(\tau)$ is a stable steady state. For $\tau \geq \bar{\tau}$, the system converges to the Point of Destruction.

\footnotetext{
${ }^{13}$ This approach is related to the political-economy equilibrium and developed in e.g. (Glomm and Ravikumar 1997) and applied in (Prieur and Bréchet 2013). However, we do not solve for the optimal tax but instead show what a tax could achieve or lead to.
} 
Proof 5 Define

$$
G\left(E_{t} ; \tau\right)=\frac{\theta_{t}}{1+\theta_{t}}\left[E_{t}+\kappa \frac{E_{t}^{\alpha}}{\theta_{t}^{\alpha-1}}-\gamma \tau\right]
$$

which is strictly increasing and concave in $E$. Thus, the slope of $G\left(E_{t} ; \tau\right)$ is given by $\frac{\partial G}{\partial E}>0$. In the case where there are two steady states, that is, $\tau \in(0, \bar{\tau})$, at the lower steady state, $G(E ; \tau)$ must cross the 45 -degree line from below, since $G(0 ; \tau)<0$. Thus, $\frac{\partial G\left(E^{l}(\tau)\right)}{\partial E}>1$, implying that the steady state $E^{l}(\tau)$ is unstable. Similarly, at steady state $E^{h}(\tau), G(E ; \tau)$ must cross the 45 -degree line from above, that is, $\frac{\partial G\left(E^{h}(\tau)\right)}{\partial E}<1$. Combining this with $\frac{\partial G}{\partial E}>0$, we conclude that the steady state $E^{h}(\tau)$ is stable.

If a policy maker introduces a lump-sum tax, then this thus has the consequence of introducing a trap. For too low wages, or too high taxes, a given lump-sum tax will lead to convergence to the Point of Destruction for $E_{0}<E^{l}(\tau)$. As a result, the danger associated with this policy may outweigh the benefits if there is uncertainty around the effectiveness of the policy and the level of environmental quality relative to $E^{l}(\tau)$.

Corollary 2 The steady states under the lump-sum tax are ordered according to $E^{l}(\tau)<$ $E^{w}(\bar{\tau})<E^{h}(\tau)<E_{H}$, for any $\tau \in(0, \bar{\tau})$, while $E_{L}<E^{h}(\tau)$ if $\tau<\left(\left(\theta_{L} / \theta_{H}\right)^{\alpha}-\theta_{L} / \theta_{H}\right) \frac{\kappa^{\frac{1}{1-\alpha}}}{\gamma}$. Also, $E_{L}<E^{l}(\tau)$ if $\tau>\left(\left(\theta_{L} / \theta_{H}\right)^{\alpha}-\theta_{L} / \theta_{H}\right) \frac{\kappa^{\frac{1}{1-\alpha}}}{\gamma}$ and $\theta_{H} / \theta_{L}>\alpha^{\frac{1}{\alpha-1}}$. Else, $E_{L}>E^{h}(\tau)$.

Proof 6 We define $\Gamma\left(E_{j}\right) \equiv \kappa \theta_{H}^{1-\alpha} E_{j}^{\alpha}-\gamma \theta_{H} \tau-E_{j}$, for $j=l$,h. Then evaluating $\Gamma\left(E_{j}\right)$ at $E_{L}$ and solving for $\tau$ gives the desired results. The last condition comes from noticing that whether the slope of $\kappa \theta_{H}^{1-\alpha} E_{j}^{\alpha}-\gamma \theta_{H} \tau$ is greater or smaller than one when evaluated at $E_{L}$ implies whether $E_{L}$ is smaller than $E^{l}(\tau)$ or larger than $E^{h}(\tau)$.

Consequently, for a sufficiently high $\theta_{H} / \theta_{L}$ ratio, we may have the result that $E_{L}<E^{l}(\tau)$. In this case a policy that raises a lump-sum tax when environmental quality is below $E^{l}(\tau)$, or $E_{t}<E^{l}(\tau)$, in order to shift the threshold above $E^{l}(\tau)$, will induce convergence to the Point of Destruction based on Proposition 5. As a result, this policy should only be applied when environmental quality is sufficiently high, such that the stable steady state $E^{h}(\tau)$ will be approached. 
If we take the high steady state $E^{h}(\tau)$, then consumption is given by

$$
c^{h}=\alpha\left(k^{h}\right)^{\alpha}=\frac{\alpha}{\gamma^{\alpha}} \frac{\left(E^{h}\right)^{\alpha}}{\theta_{H}^{\alpha}} .
$$

Thus the steady state utility is $U^{h}=\ln \left(c^{h}\right)+\theta_{H} \ln \left(E^{h}\right)$ and the willingness to pay to shift $\widehat{E}$ to be above $E^{h}$ is given by

$$
\Lambda(\tau)=U^{h}-U_{L}=\ln \left(\frac{\alpha}{\gamma^{\alpha} \theta_{H}^{\alpha}}\right)+\left(\alpha+\theta_{H}\right) \ln \left(E^{h}\right)-U_{L},
$$

where $U_{L}$ is independent of $\tau$.

This gives

$$
\Lambda(0)=U_{H}-U_{L}>0, \quad \frac{\partial \Lambda(\tau)}{\partial \tau}=\left(\alpha+\theta_{H}\right) \frac{\partial E^{h}}{\partial \tau}<0 .
$$

Thus, there exists a $\underline{\tau}>0$, such that $\Lambda(\underline{\tau})=0$.

As a result, the maximum willingness to pay to shift $\widehat{E}>E^{h}$ is given by $\tau^{L S}=\underline{\tau}$, for $\tau^{L S}<\bar{\tau}$. Though the arguments are similar to the above income-tax case, the results differ.

Conclusively, when we thus look at our three potential policies that may shift the threshold (costless, percent income tax or lump-sum tax), we have shown that each has its own advantages and limitations. We have also argued that, in terms of policy choice, it is important to know which mechanism leads to the existence of the threshold. In some cases the threshold is more likely to occur through government regulation, like it is the case for ozone standards. In other cases, like the attitude towards nuclear energy, thresholds are more likely to occur due to cultural dynamics or median voter outcomes.

\section{Conclusion}

In this article we studied the implication of thresholds in preferences. To model this we extended the basic model of John and Pecchenino by allowing the current level of environmental quality to have a discrete impact on how an agent trades off future consumption and environmental quality. We motivate the existence of the threshold based 
on research from political science, from arguments based on regulation and standards, cultural economics as well as ecological economics.

Our results are that the location of the threshold determines both the potential steady states as well as the dynamics. For low thresholds, as it would be the case in consumptionoriented societies, we find that environmental quality converges to a low steady state. In contrast, if the threshold is sufficiently high, which would hold in postmaterialistic societies, then environmental quality converges to a high steady state. For intermediate levels of the threshold we find that environmental quality converges to a stable p-cycle, with environmental quality being asymptotically bounded below and above by the low and high steady state. This thus has implications for intergenerational equity, since we can show that, even in the near future, standard criteria of intergenerational equity are violated.

We then draw several policy implications at steady state. Importantly, we discuss under which circumstances policy interventions are useful despite this endogeneity in preferences. The policy intervention that we look at is a shift in the threshold. Our criteria for a potential policy intervention is that, if each individual in every potential state of the world would be better off by the policy intervention, then the policy should be undertaken. Our results are that, if it is costless to shift the threshold, then it is always worthwhile to do so. This has implications for the setting of environmental standards. In case a standard needs to be imposed, our results indicate that it is always better to set the standard at a higher level than a lower one. This, obviously, rests on the assumption that it is costless to set the standard. In contrast, if it is costly to change the threshold, e.g. in case educational measures need to be undertaken, then it is worthwhile to change the threshold if the threshold originally was very low. In that case, it is certainly important to know more precisely about the costs and benefits involved in changing the threshold, and the policy would be case-specific. For example, we have shown that in the case of a lump-sum tax, a policy maker could essentially create a development trap. The results in this article suggest that only richer societies should consider applying a lump-sum tax since otherwise they may be caught in the development trap. 
In terms of future research, we suggest to extend this work to a long-term planner. This is difficult in so far as the modeling in this case is not clear. Firstly, one would imagine that an infinitely-lived planner sets the threshold high enough so that convergence to the steady state is assured. In this case, our previous results are unlikely to carry forward to the infinitely-lived planner case. However, one could also imagine that the planner cannot choose the threshold, e.g. if it arises due to cultural dynamics that he views outside of his control. In this case the change in preferences whenever environmental quality crosses the threshold is difficult to deal with analytically. Consequently, whether the results from our article carry forward to the infinitely-lived planner case depends on how much power or freedom one gives to the planner. 


\section{References}

Bisin, A. and T. Verdier, "On the cultural transmission of preferences for social status," Journal of Public Economics, 1998, 70 (1), 75-97.

Bisin, Alberto and Thierry Verdier, "The economics of cultural transmission and the dynamics of preferences," Journal of Economic Theory, 2001, 97 (2), 298-319.

Bowles, Samuel, "Endogenous preferences: The cultural consequences of markets and other economic institutions," Journal of economic literature, 1998, pp. 75-111.

Campbell, D., W.G. Hutchinson, and R. Scarpa, "Incorporating discontinuous preferences into the analysis of discrete choice experiments," Environmental and resource economics, 2008, 41 (3), 401-417.

Croix, D. De La and P. Michel, A theory of economic growth: dynamics and policy in overlapping generations, Cambridge University Press, 2002.

Downs, A., "Up and down with ecology: The issue attention cycle," Public interest, 1972, 28 (1), 38-50.

Erskine, H., "The polls: Pollution and its costs," The Public Opinion Quarterly, 1972, $36(1), 120-135$.

Gelso, Brett R and Jeffrey M Peterson, "The influence of ethical attitudes on the demand for environmental recreation: incorporating lexicographic preferences," Ecological Economics, 2005, 53 (1), 35-45.

Georgescu-Roegen, N., "Choice, expectations and measurability," The Quarterly Journal of Economics, 1954, pp. 503-534.

Glomm, Gerhard and Balasubramanian Ravikumar, "Productive government expenditures and long-run growth," Journal of Economic Dynamics and Control, 1997, 21 (1), 183-204. 
Goulao, C. and A. Pérez-Barahona, "Intergenerational transmission of noncommunicable chronic diseases," Journal of Public Economic Theory, forthcoming, 2013.

Gowdy, J.M., "The value of biodiversity: markets, society, and ecosystems," Land Economics, 1997, pp. 25-41.

Graumann, C.F. and L. Kruse, The environment: Social construction and psychological problems, Sage Publications, Inc, 1990.

John, A. and R. Pecchenino, "An overlapping generations model of growth and the environment," The Economic Journal, 1994, pp. 1393-1410.

Jouvet, P.A., P. Michel, and G. Rotillon, "Optimal growth with pollution: how to use pollution permits?," Journal of Economic Dynamics and Control, 2005, 29 (9), 1597-1609.

Jr, Harl E Ryder and Geoffrey M Heal, "Optimal growth with intertemporally dependent preferences," The Review of Economic Studies, 1973, pp. 1-31.

Kennedy, S.E., The banking crisis of 1933, University Press of Kentucky Lexington, 1973.

Lee, S., I. Schumacher et al., "When does financial sector (in) stability induce financial reforms?," Ecole Polytechnique Paris Working Paper Series no. 2011-21, 2011.

Linton, R., Acculturation in seven American Indian tribes, Peter Smith Pub Inc., 1963 (1940 reprint).

Lockwood, M., "Non-compensatory preference structures in non-market valuation of natural area policy," Australian Journal of Agricultural Economics, 1996, 40 (02).

Marschak, TA, "On the study of taste changing policies," The American Economic Review, 1978, pp. 386-391. 
Olli, E., G. Grendstad, and D. Wollebaek, "Correlates of environmental behaviors," Environment and Behavior, 2001, 33 (2), 181-208.

Prieur, Fabien and Thierry Bréchet, "Can education be good for both growth and the environment?," Macroeconomic Dynamics, 2013, pp. 1-23.

Schumacher, I., "The dynamics of Environmentalism and the Environment," Ecological Economics, 2009, 68 (11), 2842-2849.

and B. Zou, "Pollution perception: A challenge for intergenerational equity," Journal of Environmental Economics and Management, 2008, 55 (3), 296-309.

Sen, Amartya K, Collective choice and social welfare, North-Holland Publishing Co., 1970.

Spash, C.L., "Ecosystems, contingent valuation and ethics: the case of wetland recreation," Ecological Economics, 2000, 34 (2), 195-215.

Stevens, T.H., J. Echeverria, R.J. Glass, T. Hager, and T.A. More, "Measuring the existence value of wildlife: what do CVM estimates really show?," Land Economics, 1991, 67 (4), 390-400.

Villacorta, M., R. Koestner, and N. Lekes, "Further validation of the motivation toward the environment scale," Environment and Behavior, 2003, 35 (4), 486-505.

Wan, Henry Y, "Optimal saving programs under intertemporally dependent preferences," International Economic Review, 1970, 11 (3), 521-547.

\section{A Appendix}

\section{A.1 Proof of Proposition 4.}

To understand the idea and logic of the proof, we start with the 2 -cycle, then we finish the proof with the general $p$-cycle. 
Step 1. Suppose that $\theta_{L} \kappa^{\frac{1}{1-\alpha}}<\widehat{E}<\theta_{H} \kappa^{\frac{1}{1-\alpha}}$ and suppose that a 2-cycle appears, then it follows that

$$
E_{L}<E_{H L}<\hat{E}<E_{L H}<E_{H} .
$$

Proof. From the definition of 2-cycle, we have

$$
\begin{aligned}
& E_{L H}=\frac{\theta_{H}}{1+\theta_{H}}\left(E_{H L}+\kappa\left(\frac{E_{H L}}{\theta_{L}}\right)^{\alpha}\right), \\
& E_{H L}=\frac{\theta_{L}}{1+\theta_{L}}\left(E_{L H}+\kappa\left(\frac{E_{L H}}{\theta_{H}}\right)^{\alpha}\right) .
\end{aligned}
$$

Substituting $E_{H L}$ into eq. (20) gives

$$
E_{L H}=\frac{\theta_{H}}{1+\theta_{H}}\left[\frac{\theta_{L}}{1+\theta_{L}}\left(E_{L H}+\kappa\left(\frac{E_{L H}}{\theta_{H}}\right)^{\alpha}\right)+\frac{\kappa}{\theta_{L}^{\alpha}}\left(\frac{\theta_{L}}{1+\theta_{L}}\left(E_{L H}+\kappa\left(\frac{E_{L H}}{\theta_{H}}\right)^{\alpha}\right)^{\alpha}\right] \equiv \Omega\left(E_{L H}\right) .\right.
$$

The left-hand side is a linear function in $E_{L H}$, while the right-hand side is a concave function starting at zero. The solution to this equation is where the linear left-hand side crosses the concave right-hand side. This defines $E_{L H}$. Then if $\Omega\left(E_{H}\right)-E_{H}<0$, we know that $E_{H}>E_{L H}$. Thus, substituting $E_{H}$ into the equation above gives, after simplifying, the condition

$$
-\frac{1}{1+\theta_{L}}\left[1-\frac{\left(1+\theta_{L}\right)^{\alpha-1}}{\left(1+\theta_{H}\right)^{\alpha-1}}\right]<0 .
$$

This holds under assumption $\theta_{H}>\theta_{L}$.

The proof for $E_{H L}>E_{L}$ proceeds along the same line.

Step 2. Now let us demonstrate that this is actually true for any $p$-cycle.

Indeed, for any $p$-cycle, if $E_{t}>\widehat{E}$, then eventually we have that $E_{t+\tau}<\widehat{E}$ for some $\tau \geq 1$. At that moment the preferences change and eventually, we will have $E_{t+\tau+i}>\widehat{E}$ for some $i \geq 1$. The question is whether $E_{t+\tau+i}>E_{H}$. We shall consider different cases based on the difference equation (7).

Case I. $E_{t}<\widehat{E}$ and $E_{t-1}<\widehat{E}$ while $E_{t+1}>\widehat{E}$.

In this case, $\theta_{t-1}=\theta_{H}$ and $\theta_{t}=\theta_{H}$, thus, the difference equation (7) is

$$
E_{t+1}=\frac{\theta_{H}}{1+\theta_{H}}\left[E_{t}+\kappa\left(\frac{E_{t}}{\theta_{H}}\right)^{\alpha}\right] \text {. }
$$


Given the assumption that $E_{t+1}>\widehat{E}$, we could take $x E_{t+1}=E_{t}$ with $0<x<1$ and hence,

$$
E_{t+1}=\frac{\theta_{H}}{1+\theta_{H}}\left[x E_{t+1}+\kappa\left(\frac{x E_{t+1}}{\theta_{H}}\right)^{\alpha}\right] \equiv \psi\left(E_{t+1}\right) .
$$

If we can prove that $\psi\left(E_{H}\right)-E_{H}<0$, then we must have $E_{t+1}<E_{H}$, given $E_{t+1}$ is the unique solution of the difference equation where the right hand side is strictly concave in $E_{t+1}$.

It is easy to check that $\psi\left(E_{H}\right)<E_{H}$ if and only if

$$
x+\frac{\kappa x^{\alpha} E_{H}^{\alpha-1}}{\theta_{H}^{\alpha-1}}<1+\frac{1}{\theta_{H}}
$$

which is equivalent to

$$
x+\frac{x^{\alpha}}{\theta_{H}}<1+\frac{1}{\theta_{H}} .
$$

The last inequality is always true due to the fact that $0<x<1$.

Case II $E_{t-1}<\widehat{E}, E_{t}>\widehat{E}$ and $E_{t+1}>\widehat{E}$.

In this case $\theta_{t-1}=\theta_{H}$ while $\theta_{t}=\theta_{L}$, thus the difference equation at $t+1$ is

$$
E_{t+1}=\frac{\theta_{L}}{1+\theta_{L}}\left[E_{t}+\kappa\left(\frac{E_{t}}{\theta_{H}}\right)^{\alpha}\right]<\frac{\theta_{H}}{1+\theta_{H}}\left[E_{t}+\kappa\left(\frac{E_{t}}{\theta_{H}}\right)^{\alpha}\right]<E_{H},
$$

where the first inequality is strict since $\theta_{L}<\theta_{H}$ and the last inequality comes from Case I.

Case III $E_{t-1}>\widehat{E}, E_{t}>\widehat{E}$ and $E_{t+1}>\widehat{E}$.

In this situation, we have $\theta_{t-1}=\theta_{t}=\theta_{L}$. Thus we get the difference equation

$$
E_{t+1}=\frac{\theta_{L}}{1+\theta_{L}}\left[E_{t}+\kappa\left(\frac{E_{t}}{\theta_{L}}\right)^{\alpha}\right]
$$

(III.a) If $E_{t} \leq E_{t+1}$, assume $x E_{t+1}=E_{t}$ with $0<x<1$. Then, the above difference equation is

$$
E_{t+1}=\frac{\theta_{L}}{1+\theta_{L}}\left[x E_{t+1}+\kappa\left(\frac{x E_{t+1}}{\theta_{L}}\right)^{\alpha}\right] \equiv \psi\left(E_{t+1}\right)
$$


Similar to the Case I arguments that if we can prove that $\psi\left(E_{H}\right)-E_{H}<0$, then we must have $E_{t+1}<E_{H}$.

It is easy to check that $\psi\left(E_{H}\right)<E_{H}$ if and only if

$$
\frac{\theta_{L}}{1+\theta_{L}}\left[x \theta_{H}+\frac{x^{\alpha} \theta_{H}^{\alpha}}{\theta_{L}^{\alpha}}\right]<\theta_{H},
$$

which is equivalent to

$$
x \theta_{L}+\frac{x^{\alpha} \theta_{H}^{\alpha-1}}{\theta_{L}^{\alpha-1}}<1+\theta_{L} .
$$

The last inequality is always true due to the fact that $\theta_{L}<\theta_{H}$ and $0<x<1$.

(III.b) Suppose $E_{t}>E_{t+1}>\widehat{E}$.

Then we only need to consider the upper bound of $E_{t}$. Apply the same arguments as in Case I and Case II for $E_{t}$, we can show that $E_{t}<E_{H}$, except in the case where $E_{t-2}>\widehat{E}$ and $E_{t-1}>\widehat{E}$ and $E_{t-1}>E_{t}$; then we consider $E_{t-1}$, and continue this process until at one step $t-\tau+1$ for some $\tau \geq 1$ and where $E_{t-\tau-1}<\widehat{E}$ and $E_{t-\tau}>\widehat{E}$. However, this is Case II and we have shown $E_{t-\tau+1}<E_{H}$. Hence, $E_{t-\tau+2}<E_{t-\tau+1}<E_{H}$, and so on, we have $E_{t}<E_{H}$.

That completes the proof. 